\title{
CME im Allergo Journal
}

$\mathrm{F}$ ortbildung - für Mediziner schon immer eine Selbstverständlichkeit, bislang vorwiegend auf freiwilliger Basis. Vor dem Hintergrund entsprechender amerikanischer und europäischer Aktivitäten hat sich in den letzten Jahren auch in Deutschland die Politik des Themas angenommen, mit dem Ziel einer verpflichtenden Fortbildung für jeden Arzt. Fortbildung fand in der Vergangenheit meist frontal in abgedunkelten Sälen statt und nicht selten stieß Morpheus dabei auf dankbare Anhänger. Um das Angebot zu verbreitern und jedem Kollegen die Möglichkeit zu einer ausreichenden Fortbildung zu geben, wird zunehmend Fortbildung von den Printmedien angeboten, nunmehr auch vom Allergo Journal. Die Abkürzung CME steht dabei für „Continuing Medical Education", auf Deutsch einfacher „zertifizierte Fortbildung".

Die Fortbildungspflicht für Ärzte ist seit Januar 2004 nicht mehr nur in der Berufsordnung und den Kammergesetzen geregelt. Nach Inkrafttreten des Gesundheits-Modernisierungs-Gesetzes ist der Nachweis der ärztlichen Fortbildung seit dem 1. Juli 2004 gesetzlich festgeschriebene Pflicht $(\$ 95$ d SGB V): „Der Vertragsarzt ist verpflichtet, sich in dem Umfang fachlich fortzubilden, wie es zur Erhaltung und Fortentwicklung der zu seiner Berufsausübung in der vertragsärztlichen Versorgung erforderlichen Fachkenntnisse notwendig ist. "Aufgabe der Ärztekammern ist es, die Qualität der Fortbildungsangebote zu prüfen und den Teilnehmern Fortbildungspunkte zu bescheinigen. Eine Mustersatzungsregelung der Fortbildung wurde auf dem 107. Deutschen Ärztetag am 20. Mai 2004 beschlossen und ist im Internet ab-

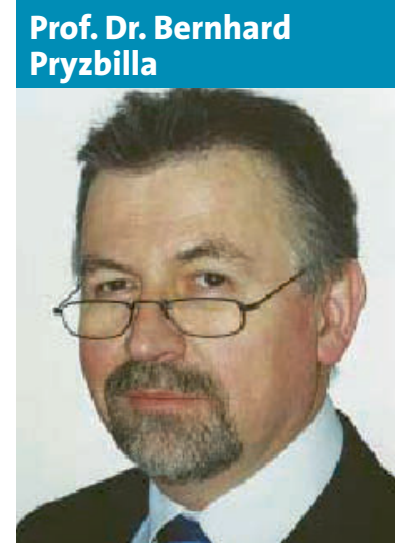

Klinik und Poliklinik für Dermatologie und Allergologie, Klinikum der Ludwig-MaximiliansUniversität München
Wunsch nach Weiterentwicklung und Verfestigung der ärztlichen Kenntnisse und Fähigkeiten entsprechen sowie den sich aus der Entwicklung von Medizin und Epidemiologie ableitenden Fortbildungsbedarf" berücksichtigen.

Generell gilt auch für Krankenhausärzte die neue Fortbildungspflicht. Bislang sind jedoch sind weder der Umfang noch die Sanktionen bei nicht erfüllter Pflicht festgeschrieben oder beschlossen.

Bislang ungelöstes Kernproblem ist die Frage nach der Wirksamkeit von Fortbildungsmaßnahmen: Nach An- rufbar: www.bundesaerztekammer.de/ 30/Fortbildung/50FbNachweis/index. html.

Alle 5 Jahre muss ein Vertragsarzt seiner KV den Erwerb von 250 Fortbildungspunkten nachweisen. Erster Stichtag für Ärzte, die am 30. Juni 2004 zugelassen waren, ist der 30. Juni 2009. Punkte, die bereits jetzt gesammelt wurden, können für diese erste 5-Jahres-
det werden. Nach heutigem Stand wird das Honorar des Vertragsarztes zunächst für ein Jahr um $10 \%$ gekürzt, sofern die vorgeschriebene Punktzahl nicht erreicht wird; weitere Kürzungen und ggf. der Entzug der Zulassung sind die nächsten Sanktionen.

Generell besteht keine Pflicht, bestimmte Themen in einem Fach abzudecken. Laut Bundesärztekammer soll die Fortbildung den „,individuellen Bedürfnisse[n] des Arztes, die dem persönlichen

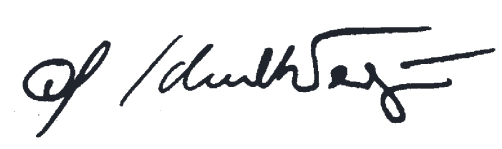

Prof. Dr. G. Schultze-Werninghaus gaben der Bundesärztekammer liegen überzeugende Erkenntnisse vor, nach denen die am häufigsten genutzten Fortbildungsformen - insbesondere solche im Vorlesungsstil - ärztliches Verhalten verhältnismäßig wenig ändern: „Diese Form der Fortbildung nimmt zwar Einfluss auf die ärztliche Kompetenz, also auf Wissen, Kenntnisse und Einstellungen, seltener aber auf das ärztliche Verhalten. Effizientes Lernen sollte deshalb durch eine sinnvolle Evaluationsmethode unterstützt werden. Sie sollte auch in der Lage sein, dem Lernenden zu helfen, seinen Lernbedarf problemorientiert einzuschätzen. Die Motivation des Lernenden ist entscheidend für den Lernerfolg."

Herausgeber und Verlag hoffen, dass Sie aus der zertifizierten Fortbildung im Allergo Journal größtmöglichen Nutzen ziehen werden (S. 397)! Herrn Priv.-Doz. Dr. Kleine-Tebbe danken wir für sein großes Engagement für CME im Allergo Journal.

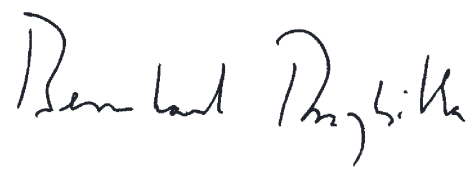

Prof. Dr. B. Pryzbilla 